\title{
CHOLERA IN BRUNEI
}

\author{
Major A. H. DIMOND, \\ M.B., B.Ch., M.R.C.P., D.T.M. \& H., R.A.M.C.
}

\section{Introduction}

SINCE 1961 there has been a change in the distribution of the incidence of cholera (S. Mukerjee et al, 1964). The El Tor vibrio has begun to spread from its normal endemic location in the Celebes to other countries in the Far East; to India where it tends to displace the classical vibrio from its position as the causative organism of the disease; and to Iran and Bahrein. The higher carrier rate in El Tor infections makes the dissemination of this vibrio more likely in the future.

I was given the chance of assisting in the recent civilian outbreak in Brunei and believing that there are now several others of my generation who have not seen cholera I have thought it worthwhile to record a few observations.

\section{Epidemiology}

The outbreak was not a large one and was well contained. Of the 221 persons admitted, 92 had clinical cholera. There were also 22 symptomless carriers and 107 cases with gastroenteritis who probably did not have cholera. Most of the cases originated from Kampong Ayer, villages of houses on stilts in which 14,000 people live on the river around the celebrated Brunei Mosque. Here sewage is often dropped directly into the river, which is used by many for bathing and washing. Conditions are ideal for spread of the disease. Exactly how the infection originated is not known, but the city is visited by people from many countries around. At the time of the outbreak many food stalls had been set up on the padang for the Royal weddings. Many of these were served by food vendors, some of whom had come from Sarawak for the purpose, where there had been a recent outbreak of the disease.

The first case was admitted on 19th September, 1965, and confirmed bacteriologically as an El Tor infection on 21st September. The peak of the epidemic was reached between 25 th and 28 th September. By 3rd October new admissions had practially ceased.

\section{Containment Measures}

Measures to limit the outbreak were started at once. The district of Brunei was isolated in every possible way. About 90 per cent of the population of 90,000 were inoculated. Public gatherings and festivities were stopped. All stalls and coffee shops were closed and food hawkers prevented from selling their wares, resumption of trade only being permitted after full inspection by the health authority. Movement of perishable goods was limited and road blocks were set up by the police to prevent the uninoculated from wandering. Further measures included the supply of uninfected water to Kampong Ayer by water boats. Houses from which cholera cases originated were quarantined, being released only when the occupants had been shown to have negative stools on culture. Any carriers discovered were admitted to hospital. 


\section{Clinical Features and Treatment}

Of the cases admitted to hospital 78 were confirmed by stool culture. All gradations of severity were seen from those who were carriers or had mild diarrhoea to those who were desperately ill and dehydrated. These latter presented an unforgettable picture akin to the textbook description. Striking features were the apparently effortless escape of fluid orally and rectally, the intense dehydration and appearance of death contrasting with the presence of a weak but perfectly audible and intelligible whispered voice denoting sensibility and awareness of the situation by the patient. The rapid change in appearance and return to strength of the rehydrated patient within a matter of a few hours was dramatic.

Treatment was based upon a regime dictated by the physician in charge of the hospital. This was a uniform scheme for all patients but at the same time adaptable to meet the requirements of an individual case.

Severely dehydrated patients were admitted immediately to the cholera ward where intravenous infusion of normal Ringer lactate solution was started at once. This was the basic fluid used in rehydration. Specific gravity estimation of blood or plasma was performed on a few of the early cases but this was found unnecessary and time consuming and an assessment of fluid requirements was latterly made upon clinical signs alone. Of most value was the quality of the radial pulse which was usually impalpable in severe cases; secondly the skin elasticity measured by pinching the skin at the neck, on the abdomen or arm, and thirdly the level of the jugular venous pulsation. Pulse rate and blood pressure were valueless. By frequent observation of these signs a fair estimate of the time when rehydration was clinically completed could be made. The procedure was to rehydrate with normal Ringer lactate solution at the rate of approximately 1 litre every ten minutes, until clinical rehydration was achieved. During this period there seemed little risk of dangerous pulmonary oedema developing provided that the patient was propped up on a few pillows at the first sign of breathlessness or coughing. For the most part the severely dehydrated patient required from 3-6 litres of fluid run in rapidly before rehydration was achieved. Thereafter the drip rate was slowed sufficiently to maintain fluid requirements taking into account fluid lost in vomit, faeces, urine and insensible loss. Intravenous therapy was continued until loose stools had ceased, the patient was taking sufficient fluid by mouth and passing urine normally. This period was usually two to four days. Two patients had over 30 litres of intravenous fluid each.

Oral fiuids were given in the form of a mixture containing sodium bicarbonate $4 \frac{1}{2} \mathrm{G}$., potassium chloride $3 \mathrm{G}$. in $60 \mathrm{ml}$. of water four hourly as soon as this could be retained. Fruit drinks made up in $\frac{1}{2}$ normal saline were allowed freely by mouth. A fluid balance chart of a simple form was kept for each patient by the nursing staff.

Tetracycline $250 \mathrm{mgm} 6$ hourly was given routinely for 5 days, by mouth. Stemetil by intramuscular injection was found effective in the treatment of vomiting. Vasopressor or steroid drugs were not found necessary.

As soon as the patient became stronger and his faeces became normal he was given " bubor" (a local rice) for a day before starting a post dysentery type of diet which was followed a few days later by normal food. Before the patient was cleared and discharged from hospital it was essential to have three negative stool cultures, taken daily from the seventh day after admission. 


\section{Results}

Most of these patients came from the poorer part of Brunei and some were undernourished. One severely ill male patient was alleged to be 98 years old. One woman who was severely dehydrated was at term on admission and gave birth to a stillborn child a few hours later. One woman was known to have chronic nephritis, one had thyrotoxicosis and another Friedreich's ataxia. Yet there were no deaths among the patients admitted to hospital and only three developed any recognisable degree of electrolyte imbalance after rehydration. One of these was the patient with chronic nephritis, another a woman who developed oliguria and nitrogen retention which responded to appropriate therapy.

\section{Discussion}

It is interesting to note the effective containment of this epidemic. Vaccination of the majority of the population was performed early and it appeared that those who were admitted a few days after vaccination did not have as serious a form of the disease as the unvaccinated. The recent trial in the Phillippines (Philippines Cholera Committee 1965) suggests that the protection offered by this means is only of limited value. It seems-more probable that the other public health measures taken were of greater significance.

The excellent results were achieved through a well organised and executed plan of treatment carried out by good staff. Tetracycline has been shown by workers from the Pakistan SEATO research laboratory (Greenough et al, 1964) to eliminate bacteria from the stool, shorten the duration of diarrhoea and reduce the requirement of intravenous fluids. This no doubt has played a part in the better results in the treatment of this disease in recent outbreaks.

\section{Acknowledgement}

My thanks are due to Dato Dr. P. I. Franks and Dr. P. Hart, M.D., M.R.C.P., for allowing me access to the detail contained in this paper, for their advice and for allowing me to publish it.

\section{REFERENCES}

Greenough, W. B., Gordon, R. S., Rosenberg, I. S., Davies, B. I. and Benenson, A. S. (1964): Tetra. cycline in the Treatment of Cholera. Lancet. 1, 355.

MukerJeE, S.; Basu, S. and BhatTaChaRYA, P. (1965). A New Trend in Cholera Epidemiology. Brit. Med.J. 2, 837.

PhiliPPINes CHOLERA COMMITTEe. (1965). Bull. World Health Organization. 32, 603.

\section{Order of St. John of Jerusalem}

Recent appointments include:

As Officer brother - Lieutentant-Colonel. N. W. J. England, R.A.M.C. 